Published in final edited form as:

Infect Control Hosp Epidemiol. 2015 November ; 36(11): 1318-1323. doi:10.1017/ice.2015.187.

\title{
In the Endemic Setting, Clostridium difficile Ribotype 027 Is Virulent But Not Hypervirulent
}

\author{
Samuel L. Aitken, PharmD ${ }^{1,2, a, b}$, M. Jahangir Alam, PhD ${ }^{1, a}$, Mohammed Khaleduzzaman, \\ PhD $^{1}{ }^{*}$, Seth T. Walk, PhD ${ }^{3}$, William L. Musick, PharmD ${ }^{2}$, Vy P. Pham, PharmD ${ }^{4}$, Jennifer L. \\ Christensen, PharmD ${ }^{5}$, Robert L. Atmar, MD $^{6}$, Yang Xie, PhD $^{7}$, and Kevin W. Garey, \\ PharmD, MS ${ }^{1}$ \\ ${ }^{1}$ Department of Pharmacy Practice and Translational Research, University of Houston College of \\ Pharmacy, Houston, Texas \\ ${ }^{2}$ Department of Pharmacy, Houston Methodist Hospital, Houston, Texas \\ ${ }^{3}$ Department of Microbiology and Immunology, Montana State University, Bozeman, Montana \\ ${ }^{4}$ Department of Pharmacy, Memorial Hermann Northwest Hospital, Houston, Texas \\ ${ }^{5}$ Department of Internal Medicine, Baylor College of Medicine Houston, Texas \\ ${ }^{6}$ Department of Medicine, Section of Infectious Disease, Baylor College of Medicine Houston, \\ Texas \\ ${ }^{7}$ Merck \& Co., Whitehouse Station, New Jersey
}

\begin{abstract}
BACKGROUND—Conflicting reports have been published on the association between Clostridium difficile ribotypes and severe disease outcomes in patients with $C$. difficile infection (CDI); several so-called hypervirulent ribotypes have been described. We performed a multicenter study to assess severe disease presentation and severe outcomes among CDI patients infected with different ribotypes.
\end{abstract}

METHODS-Stool samples that tested positive for $C$. difficile toxin were collected and cultured from patients who presented to any of 7 different hospitals in Houston, Texas (2011-2013). $C$. difficile was characterized using a fluorescent PCR ribotyping method. Medical records were reviewed to determine clinical characteristics and ribotype association with severe CDI presentation (ie, leukocytosis and/or hypoalbuminemia) and severe CDI outcomes (ie, ICU admission, ileus, toxic megacolon, colectomy, and/or in-hospital death).

Address correspondence to Kevin W. Garey, PharmD, MS, Professor and Chair, University of Houston College of Pharmacy, 1441 Moursund St, Houston, TX 77030, (kgarey@uh.edu).

These authors contributed equally to this article.

bresent affiliation: Division of Pharmacy, The University of Texas MD Anderson Cancer Center; Houston, Texas.

*Author's name has been corrected since original publication. An erratum notice detailing this change was also published (DOI 10.1017/ice.2015.305).

Potential conflicts of interest. KWG reports funding from Merck, Cubist, and Summit pharmaceuticals companies. YX is an employee of Merck. 
RESULTS-Our study included 715 patients aged $61 \pm 18$ years (female: 63\%; median Charlson comorbidity index: $2.5 \pm 2.4$; hospital-onset CDI: $45 \%$; severe CDI: $36.7 \%$; severe CDI outcomes: $12.3 \%$ ). The most common ribotypes were 027, 014-020, FP311, 002, 078-126, and 001. Ribotype 027 was a significant independent predictor of severe disease (adjusted odds ratio [aOR], 2.24; 95\% confidence interval $[\mathrm{CI}], 1.53-3.29 ; P<.001)$ and severe CDI outcomes (aOR, 1.71; 95\% CI, $1.02-2.85 ; P=.041)$ compared with all other ribotypes in aggregate. However, in an analysis using all common ribotypes as individual variables, ribotype 027 was not associated with severe CDI outcomes more often than other ribotypes.

CONCLUSION-Ribotype 027 showed virulence equal to that of other ribotypes identified in this endemic setting. Clinical severity markers of CDI may be more predictive of severe CDI outcomes than a particular ribotype.

Clostridium difficile is the most common cause of hospital-acquired infections in the United States. ${ }^{1}$ The incidence of $C$. difficile infection (CDI) increased dramatically beginning in 2001, coinciding with the emergence of the epidemic ribotype 027 (R027, also known as North American pulsed field type 1 [NAP1] or restriction endonuclease group BI strain). ${ }^{2,3}$ Since this time, the R027 strain has spread worldwide and is now the most common ribotype reported in the United States. ${ }^{4,5}$ The high mortality rate associated with R027 led to it being described as a hypervirulent strain. Since that characterization, additional ribotypes of $C$. difficile have been described as hypervirulent using similar criteria. ${ }^{6,7}$

Previous attempts to correlate severe $C$. difficile infection (CDI) and/or patient outcomes with the strain ribotype yielded conflicting results. ${ }^{5,8-10}$ These studies differed in design and patient population and did not always account for disease treatment characteristics.

Additionally, many studies focusing on R027 were performed in an epidemic setting in which R027 accounted for the vast majority of isolates. Therefore, the impact of $C$. difficile ribotype on disease outcomes remains unclear, especially in endemic (rather than epidemic) settings. We hypothesized that patients infected with certain $C$. difficile ribotypes would experience more severe disease presentation and would be associated with epidemiologically defined severe disease outcomes. To test this hypothesis, we performed a multicenter study across 7 hospitals serving the Houston, Texas, area. The objectives of this study were to assess disease severity at presentation and outcomes of patients with CDI infected with multiple different ribotypes in an endemic setting. Finally, we assessed whether severe CDI presentation or specific ribotypes would better predict severe CDI outcomes.

\section{METHODS}

\section{Prospective Sample Collection and Microbiologic Analysis}

This multicenter cohort study was conducted in 2 healthcare systems in Houston, Texas, between 2011 and 2013. A total of 7 hospitals served as study sites, including 3 universityaffiliated tertiary care centers and 4 community hospitals. In this study, stool specimens from all patients with a stool test positive for $C$. difficile as part of routine clinical care were collected for further analysis by a centralized research microbiology laboratory. Enzymelinked immunoassay or a polymerase-chain reaction (PCR) detection of the $t c d B$ gene in 
unformed stool was the diagnostic methodology used at all study sites during the study period. C. difficile toxin-positive stool samples were plated onto cefoxitin-cycloserinefructose agar (CCFA) plates and incubated under strict anaerobic conditions for 48-72 hours. The growth of toxigenic $C$. difficile was confirmed using multiplex PCR to determine the presence of $t c d A, t c d B$, and binary toxin genes. Fluorescent ribotyping was performed as previously described. ${ }^{11}$ This technique does not distinguish between ribotypes 053 and 163, ribotypes 014 and 020 , or ribotypes 078 and 126; therefore, these are reported as combined ribotypes (ie, 053-163, 014-020, and 078-126).

\section{Data Collection and Study End Points}

Medical data were abstracted from the electronic health records of each institution. Patient and disease characteristics collected included white blood cell count (WBC) and albumin level at time of diagnostic sample, comorbid disease states (reported as the Charlson comorbidity index ${ }^{12}$ ), solid organ transplantation status, disease recurrence status (defined as medical record documentation or a positive toxin test within the 60 days preceding the index culture), initial CDI treatment (defined as the initial CDI-directed treatment used for $\geq 24$ hours following the diagnostic test), continued non-CDI antibiotic use for $\geq 24$ hours following initiation of treatment for CDI, other medication use, and standard CDI epidemiologic classification definitions. ${ }^{13}$ Only data from the first episode for which ribotyping data were available were used in patients with multiple CDI episodes.

Severe disease presentation was categorized as severe leukocytosis (WBC $\geq 15,000$ cells $/ \mu \mathrm{L}$ within 24 hours of diagnostic sample) and/or severe hypoalbuminemia (albumin $\_.5 \mathrm{~g} / \mathrm{dL}$ within 48 hours of the diagnostic sample) based on markers of severe disease identified in Walk, et al. ${ }^{8}$ As the majority of community-onset cases were expected to be missing baseline serum creatinine values, change in serum creatinine was not included as a potential severity marker. ${ }^{14}$ Severe CDI outcome was defined as CDI-attributable ICU admission, CDI-attributable colectomy, toxic megacolon, pseudomembranous colitis, CDI-related ileus, or death during hospitalization. A hypervirulent ribotype was defined as a ribotype that was associated with more severe CDI outcomes than the majority of other strains.

\section{Statistical Analysis}

Baseline characteristics and potential confounders were compared using the $\chi^{2}$ test for categorical variables and Student $t$ test or Wilcoxon rank sum test for parametric and nonparametric data, respectively. Normality was assessed using the Shapiro-Wilk test. All analyses were performed using Stata v13.1 software (StataCorp LP, College Station, TX) or SAS version 9.1 (SAS Institute, Cary, NC). For creation of multivariate models, any variable with $P<.20$ from the univariate analysis was a candidate for entry into the multivariate models. Other variables were entered as described below. A 2-sided $P<.05$ was considered statistically significant.

To assess the association between R027, severe CDI presentation, and severe CDI outcomes, separate forward stepwise logistic regression models were constructed. R027 was compared to all other ribotypes represented as a categorical variable. CDI treatment was included in models evaluating in-hospital mortality and severe CDI outcomes. To assess the role of 
demographic differences in patients with R027 versus non-R027 strains, a propensity-scorematched model was constructed. All measured characteristics (prior to the diagnostic test) were used to determine a predictive model of R027 versus all other ribotypes; the model was then conditioned using patient-specific characteristics to determine a propensity score. A 2:1 matched cohort (non-R027:R027) was created using a nearest-neighbor greedy matching algorithm with a caliper length equivalent to \pm 0.25 standard deviations of the propensity score. ${ }^{15}$ Receiver operating characteristics (ROC) analysis was used to confirm the categorization ability of the propensity score. A predictive model for death was then constructed in this matched cohort using exact logistic regression.

To assess the association between other ribotypes and severe CDI presentation and severe CDI outcomes, separate forward stepwise, logistic regression models were constructed. Any ribotype present in at least 15 patients in the dataset was given individual categorical variables. All of these ribotypes were eligible for inclusion in the multivariate model. CDI treatment was included in models evaluating in-hospital mortality and severe CDI outcomes.

This study was approved by the Institutional Review Board of the University of Houston and all study centers' institutional review boards.

\section{RESULTS \\ Cohort Characteristics}

A total of 715 patients aged $61 \pm 18$ years (female: $63 \%$; Charlson score: $2.5 \pm 2.4$; hospitalonset CDI: 45\%) were included in this analysis. The most common ribotypes were $027(\mathrm{n}=$ $170 ; 24 \%), 014-020(\mathrm{n}=118 ; 17 \%)$, FP311 $(\mathrm{n}=70 ; 10 \%), 002(\mathrm{n}=69 ; 10 \%), 078-126(\mathrm{n}=$ $42 ; 6 \%)$, and 001 and FP324 ( $\mathrm{n}=35$ each; 5\%). The full distribution of ribotypes is shown in Figure 1. Severe CDI was present in 257 of 715 patients (35.9\%; leukocytosis: $\mathrm{n}=131$ [18.3\%]; hypoalbuminemia: $\mathrm{n}=63$ [8.8\%]; both: $\mathrm{n}=63$ [8.8\%]). Severe CDI outcomes were present in 88 of 715 patients (12.3\%; death: $\mathrm{n}=44$ [6.2\%]; ICU admission: $\mathrm{n}=54$ [7.5\%]; pseudomembranous colitis: $\mathrm{n}=3$ [0.4\%]; ileus: $\mathrm{n}=11$ [1.5\%]; toxic megacolon: $\mathrm{n}=$ $7[1 \%]$; colectomy: $\mathrm{n}=6[0.8 \%]$ ). In addition, 53 patients were treated solely as outpatients and were missing treatment data. The remaining 662 patients were most commonly treated with oral metronidazole only $(\mathrm{n}=275 ; 42 \%)$, intravenous metronidazole only $(\mathrm{n}=129$; $19 \%)$, oral vancomycin only $(\mathrm{n}=80 ; 12 \%)$, or a combination of oral vancomycin and metronidazole $(\mathrm{n}=113 ; 17 \%)$. No treatment was given in the first 24 hours for 65 patients $(10 \%)$.

\section{R027 Is a Virulent Ribotype}

Compared with all other ribotypes, patients with R027 were older ( $67.1 \pm 16.7$ years vs 59.9 \pm 18.3 years; $P<.001$ ) and had a higher median (interquartile range [IQR]) Charlson comorbidity score (3 [1-5] vs $2[0-4] ; P=.009)$. Patients with R027 were also significantly less likely to be classified as having community-associated disease $(17.3 \%$ vs $32.7 \% ; P<$. $001)$ and were significantly more likely to have recurrent disease $(15.3 \%$ vs $8.3 \% ; P=.007)$. Characteristics of patients with R027 strains compared with other strains are shown in Table 1. Severe disease was significantly more common among those with R027 than in those 
without $(54.7 \%$ vs $30.8 \% ; P<.001)$. In multivariate analysis, R027 was a significant independent predictor of severe disease compared with all other ribotypes (aOR, 2.24; 95\% CI, 1.53-3.29; $P<.001)$. Severe disease outcomes were significantly more common among patients with R027 strains than in those with other strains $(18.8 \%$ vs $10.2 \% ; P<.0028)$. In multivariate analysis, R027 was a significant independent predictor of severe CDI outcomes compared with all other ribotypes $(\mathrm{aOR}, 1.71 ; 95 \% \mathrm{CI}, 1.02-2.85 ; P=.041)$. Full results of the multivariate logistic regression models are shown in Table 2 . In the propensity-scorematched cohort, the derived propensity score was able to adequately characterize patients with R027 strains (area under the ROC curve, 0.716; 95\% CI, 0.67-0.76) and generated a total of 150 matched trios. In this cohort, R027 was a significant predictor of death (aOR, $3.34 ; 95 \%$ CI, $1.41-8.53 ; P=.005)$.

To compare our results, the model was rerun combining ribotypes 027 and 078 , similar to the study by Walk et al. ${ }^{8}$ In multivariate analysis, ribotypes 027 and 078 were significant independent predictors of severe CDI presentation (aOR, 1.69; 95\% CI, 1.18-2.41; $P=.004$ ) and severe CDI outcomes (aOR, 1.65; 95\% CI, 1.002-2.701; $P=.049$ ) compared with all other ribotypes.

\section{Other C. difficile Ribotypes Are Virulent}

The distribution of ribotypes occurring in at least 15 patients as well as the frequency of severe outcomes are presented in Table 3. In a second multivariate model, each individual ribotype was included as a categorical variable; other variables used to assess the association between different ribotypes and severe CDI presentation included age, treatment with proton pump inhibitors, treatment with narcotics, and community-acquired disease (Table 2). R027 was associated with increased incidence of severe CDI (aOR, 1.89; 95\% CI, 1.14-3.10; $P=$. 013), whereas ribotype 014-020 was associated with decreased incidence of severe CDI (aOR, 0.36; 95\% CI, 0.14-0.93; $P=.035$ ). In multivariate analysis assessing severe CDI outcomes, R027 was not associated with severe CDI outcomes more often than other virulent ribotypes, whereas ribotype 014-020 was associated with decreased incidence of severe CDI outcomes (aOR, 0.26; 95\% CI, 0.10-0.68; $P=.006)$.

\section{Severe CDI Presentation Is Predictive of Severe CDI Outcomes}

Severe CDI presentation and common ribotypes were included in a third multivariate model, along with continued use of non-CDI antibiotics and treatment with oral metronidazole (Table 2). Severe CDI presentation was associated with severe CDI outcomes (aOR, 4.19; 95\% CI, 2.46-8.07; $P<.001)$. No specific ribotype was associated with increased virulence; however, ribotype 014-020 was independently associated with decreased incidence of severe CDI outcomes (aOR, 0.34; 95\% CI, 0.13-0.91; $P<.001$ ).

\section{DISCUSSION}

In this large multicenter study, $C$. difficile R027 was the most common ribotype and was associated with severe CDI presentation and severe CDI outcomes. Although R027 was independently associated with severe disease (with R014-020 being protective), R027 was not independently associated with severe outcomes. No single strain was more virulent than 
the others. One ribotype (014-020) was associated with significantly lower incidence of severe CDI and severe CDI outcomes compared with other ribotypes. Strengths of the study include a large sample size from multiple hospitals, use of a validated ribotyping method, and multivariate analysis techniques.

These data significantly augment previous publications by Walk et al, ${ }^{8}$ Walker et al, ${ }^{9}$ and See et al. ${ }^{5}$ Walk et al evaluated 743 patients with CDI divided into a derivation and validation datasets. They used a ribotyping method identical to that used in the current study to allow direct comparison of ribotypes. In the Walk et al study, ribotype 014-020 was most prevalent, followed by ribotypes $027,053-163$, and 001 . Walk et al also considered the combination of ribotypes 027 and $078-126$ as hypervirulent based on previous reports. This hypervirulent group (027/078-126) was associated with severe CDI outcomes before adjustment for clinical severity markers (OR, 2.33; 95\% CI, 1.03-5.02) but was not associated with severe CDI outcomes after adjustment for leukocytosis or hypoalbuminemia. Walk et al concluded that this hypervirulent group does not predict severe CDI outcomes independently of clinical severity markers of disease. Our results support and confirm this finding. In addition, our result that no single ribotype predicted increased severe CDI outcomes speaks more specifically to R027 compared with the Walk et al study. See et al ${ }^{5}$ performed a population-based study of 2,057 cases of CDI using pulsed-field gel electrophoresis to type strains of $C$. difficile. Patients infected with Nap1 strains (corresponding to R027) had significantly worse outcomes than patients with Nap4 and Nap11 strains, in particular. Not controlling for severe CDI presentation, patients with Nap1 strains were more likely to experience severe CDI outcomes compared with patients who had any other strain (aOR, 1.66; 95\% CI, 1.09-2.54). This result agrees well with results from our study. The unadjusted odds ratio of severe disease in patients with Nap4 strains (corresponding with ribotype $014-020)^{16}$ was approximately 0.4 , which also agrees well with results from this study. Finally, Walker et $\mathrm{al}^{9}$ studied the relationship between $C$. difficile strain and mortality in 2,222 patients with CDI using multilocus sequence typing. Mortality rates varied between $C$. difficile types with highest mortality rates for clades corresponding to ribotypes 078,027 , and 017 and lowest mortality rates for ribotypes 023 and 014-020. These results complement very well the findings of our study.

When these findings are considered together, a number of conclusions can be posited. First, studies investigating the clinical virulence of certain strains of $C$. difficile are highly dependent on the virulence of the comparator strains. In our studies and others, ribotype 014-020 was associated with lower rates of severe CDI outcomes compared with other strains. Thus, any study that has a large proportion of 014-020 strains will have a high likelihood of demonstrating increased virulence with other strains. Due to this finding and the confusion that has ensued, we suggest that the term 'hypervirulent' be removed from the nomenclature that describes certain strains of $C$. difficile. Our second conclusion is that in hospitals with multiple virulent strains of $C$. difficile present in an endemic setting, clinical severity markers of CDI are likely more important predictors of severe CDI outcomes than any specific ribotype. We do not feel that this finding diminishes the utility of ribotyping or other strain typing method, as knowledge of strain type still provides valuable infection control information and data regarding new strain emergence, which may ultimately 
influence treatment decisions. In addition, the consistent lack of virulence for ribotype 014-020 may offer valuable insight into the pathogenesis of CDI. Finally, the differing methods used for strain typing in these studies illustrate the urgent need for a common strain typing method. Data generated using the fluorescent PCR ribotyping protocol in this study were recently shown to be highly portable between laboratories. ${ }^{17}$

Additionally, the continued use of non- $C$. difficile antibiotics was a strong predictor of severe CDI outcomes in all models. While many of the patients in our study had concomitant infections and therefore likely had a higher risk of adverse outcomes, the continued use of non- $C$. difficile antibiotics has also been associated with prolonged diarrhea and CDI treatment failure. ${ }^{18}$ Therefore, it is plausible that the continued use of antibiotics following the diagnosis of CDI increases the risk of the adverse consequences of CDI treatment failure. Second, the use of oral metronidazole was associated with a significantly lower likelihood of severe outcomes. A recently published randomized controlled trial showed that metronidazole was inferior to treatment with vancomycin at all disease severities, ${ }^{19}$ therefore, this finding likely represents residual confounding and treatment bias rather than a true efficacy signal. Regardless, the impact of both the continued use of antibiotics and treatment choice should be further studied.

Our study does have several limitations. First, as an observational study, data were not systematically collected for all patients. However, information on both the primary and secondary outcome measures were objective and universally available and therefore subject to minimal observer bias. Second, although data were available from both academic and community hospitals in the Houston area, treatment standards and patient characteristics may not reflect practices at other centers. Third, while we collected information on the treatment characteristics for patients in our study, information on concomitant disease states, such as infections or other acute illnesses, was not considered. Fourth, our sample size was not sufficient to explicitly state the expected virulence of certain ribotypes compared with others. Finally, we only collected information on the first case for which a $C$. difficile strain could be isolated, potentially biasing the results in the event that unculturable strains had differing clinical characteristics from the patients included in our study.

In conclusion, $C$. difficile ribotype 027 showed virulence equal to that of other ribotypes identified in this endemic setting. These results were largely due to a certain ribotypes (eg, R014-020) with decreased CDI disease severity and outcomes, suggesting that the comparator strains in the population being studied helps determines apparent virulence. Clinical severity markers of CDI may be more predictive of severe CDI outcomes than any particular ribotype.

\section{Acknowledgments}

Financial support. This work was supported in part by the Texas Department of State Health Services (2014-045577) and Merck \& Co., Inc. SW reports funding from NIAID (K01AI097281).

\section{References}

1. Miller BA, Chen LF, Sexton DJ, Anderson DJ. Comparison of the burdens of hospital-onset, healthcare facility-associated Clostridium difficile infection and of healthcare-associated infection 
due to methicillin-resistant Staphylococcus aureus in community hospitals. Infect Control Hosp Epidemiol. 2011; 32:387-390. [PubMed: 21460491]

2. Reveles KR, Lee GC, Boyd NK, Frei CR. The rise in Clostridium difficile infection incidence among hospitalized adults in the United States: 2001-2010. Am J Infect Control. 2014; 42:10281032. [PubMed: 25278388]

3. McDonald LC, Killgore GE, Thompson A, et al. An epidemic, toxin gene-variant strain of Clostridium difficile. New Engl J Med. 2005; 353:2433-2441. [PubMed: 16322603]

4. He M, Miyajima F, Roberts P, et al. Emergence and global spread of epidemic healthcare-associated Clostridium difficile. Nature Genet. 2013; 45:109-113. [PubMed: 23222960]

5. See I, Mu Y, Cohen J, et al. NAP1 strain type predicts outcomes from Clostridium difficile infection. Clin Infect Dis. 2014; 58:1394-1400. [PubMed: 24604900]

6. Goorhuis A, Bakker D, Corver J, et al. Emergence of Clostridium difficile infection due to a new hypervirulent strain, polymerase chain reaction ribotype 078. Clin Infect Dis. 2008; 47:1162-1170. [PubMed: 18808358]

7. Lim SK, Stuart RL, Mackin KE, et al. Emergence of a ribotype 244 strain of Clostridium difficile associated with severe disease and related to the epidemic ribotype 027 strain. Clin Infect Dis. 2014; 58:1723-1730. [PubMed: 24704722]

8. Walk ST, Micic D, Jain R, et al. Clostridium difficile ribotype does not predict severe infection. Clin Infect Dis. 2012; 55:1661-1668. [PubMed: 22972866]

9. Walker AS, Eyre DW, Wyllie DH, et al. Relationship between bacterial strain type, host biomarkers, and mortality in Clostridium difficile infection. Clin Infect Dis. 2013; 56:1589-1600. [PubMed: 23463640]

10. Miller M, Gravel D, Mulvey M, et al. Health care-associated Clostridium difficile infection in Canada: patient age and infecting strain type are highly predictive of severe outcome and mortality. Clin Infect Dis. 2010; 50:194-201. [PubMed: 20025526]

11. Alam MJ, Anu A, Walk ST, Garey KW. Investigation of potentially pathogenic Clostridium difficile contamination in household environs. Anaerobe. 2014; 27:31-33. [PubMed: 24657158]

12. Charlson ME, Pompei P, Ales KL, MacKenzie CR. A new method of classifying prognostic comorbidity in longitudinal studies: development and validation. J Chronic Dis. 1987; 40:373383. [PubMed: 3558716]

13. McDonald LC, Coignard B, Dubberke E, et al. Recommendations for surveillance of Clostridium difficile-associated disease. Infect Control Hosp Epidemiol. 2007; 28:140-145. [PubMed: 17265394]

14. Shah DN, Bhatt NS, Welch JK, Koo HL, Garey KW. Defining acute renal dysfunction as a criterion for the severity of Clostridium difficile infection in patients with community-onset vs hospital-onset infection. J Hosp Infect. 2013; 83:294-299. [PubMed: 23433867]

15. Austin PC. An introduction to propensity score methods for reducing the effects of confounding in observational studies. Multivariate Behav Res. 2011; 46:399-424. [PubMed: 21818162]

16. Tenover FC, Akerlund T, Gerding DN, et al. Comparison of strain typing results for Clostridium difficile isolates from North America. J Clin Microbiol. 2011; 49:1831-1837. [PubMed: 21389155]

17. Martinson JN, Broadaway S, Lohman E, et al. Evaluation of portability and cost of a fluorescent PCR ribotyping protocol for Clostridium difficile epidemiology. J Clin Microbiol. 2015 In press.

18. Mullane KM, Miller MA, Weiss K, et al. Efficacy of fidaxomicin versus vancomycin as therapy for Clostridium difficile infection in individuals taking concomitant antibiotics for other concurrent infections. Clin Infect Dis. 2011; 53:440-447. [PubMed: 21844027]

19. Johnson S, Louie TJ, Gerding DN, et al. Vancomycin, metronidazole, or tolevamer for Clostridium difficile infection: results from two multinational, randomized, controlled trials. Clin Infect Dis. 2014; 59:345-354. [PubMed: 24799326] 


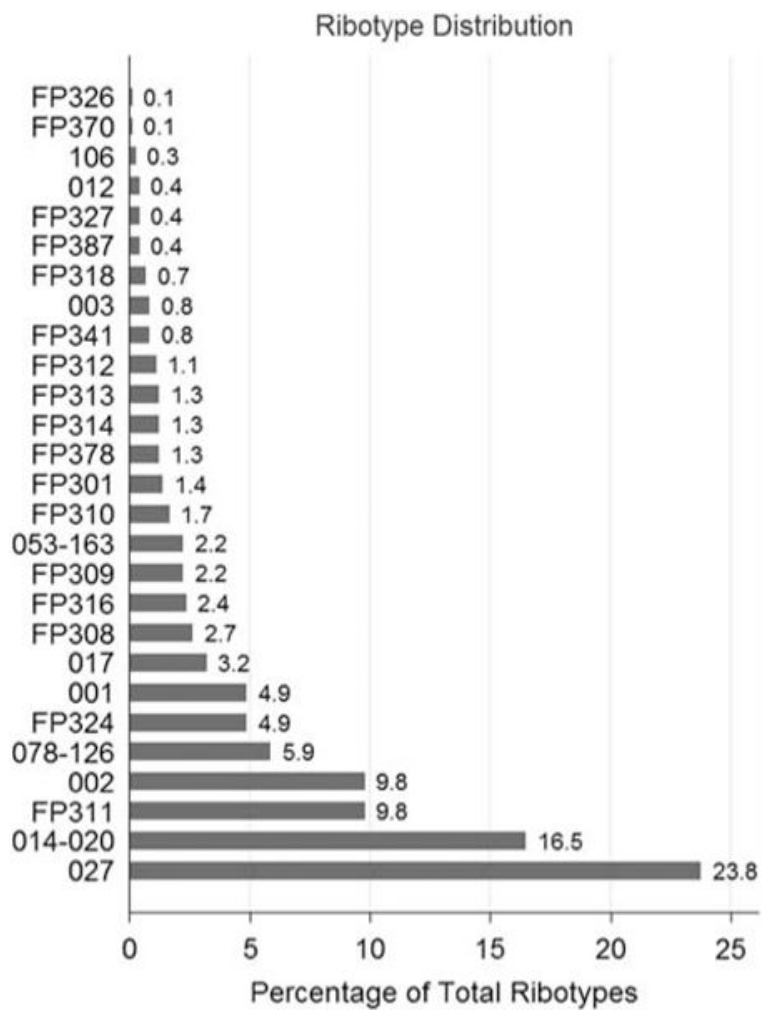

FIGURE 1.

Ribotype distribution for all isolates. 


\section{TABLE 1}

Cohort Characteristics (Ribotype (R) 027 vs Non-R027 Ribotypes) ${ }^{10}$

\begin{tabular}{|c|c|c|c|c|}
\hline Characteristic & Overall $(n=715)$ & R027 $(n=170)$ & Non-R027 $(n=545)$ & $P$ Value \\
\hline Age, y, mean (SD) & $61.6(18.2)$ & $67.1(16.7)$ & $59.9(18.3)$ & $<.001$ \\
\hline Charlson comorbidity index, median (IQR) & $2(0-4)$ & $3(1-5)$ & $2(0-4)$ & $<.001$ \\
\hline Male gender & 36.9 & 40.6 & 33.4 & .257 \\
\hline \multicolumn{5}{|l|}{ Epidemiologic characteristics } \\
\hline Community-associated & 29.0 & 17.3 & 32.7 & $<.001$ \\
\hline COHCFA & 24.5 & 22.8 & 25.0 & .568 \\
\hline HOHCFA & 44.6 & 57.7 & 40.6 & $<.001$ \\
\hline \multicolumn{5}{|l|}{ Other characteristics } \\
\hline Outpatient visit only & 9.0 & 7.1 & 9.5 & .322 \\
\hline Emergency-department visit only & 3.8 & 2.9 & 4.0 & .513 \\
\hline Recurrent disease & 9.9 & 15.3 & 8.3 & .007 \\
\hline Solid organ transplantation & 5.9 & 4.1 & 6.4 & .265 \\
\hline Prior proton pump inhibitor & 61.8 & 63.8 & 61.2 & .568 \\
\hline Chronic use of $\geq 20 \mathrm{mg}$ prednisone equivalent per day & 6.0 & 3.8 & 6.7 & .168 \\
\hline Scheduled narcotics & 9.0 & 9.1 & 11.9 & .321 \\
\hline \multicolumn{5}{|l|}{ Treatment characteristics } \\
\hline Non-C. difficile antibiotics continued $\geq 24 \mathrm{~h}$ & 53.4 & 61.3 & 50.8 & .022 \\
\hline Oral vancomycin & 21.9 & 16.1 & 23.8 & .038 \\
\hline Metronidazole (intravenous) & 29.3 & 23.5 & 31.2 & .060 \\
\hline Metronidazole (oral) & 51.4 & 43.2 & 54.0 & .017 \\
\hline Both vancomycin and metronidazole & 17.1 & 27.2 & 13.8 & $<.001$ \\
\hline
\end{tabular}

NOTE. SD, standard deviation; IQR, interquartile range; COHCFA, community-onset, healthcare facility associated; HOHCFA, healthcare-onset, healthcare facility associated. 


\section{TABLE 2}

Multivariate Regression Models Evaluating the Association Between Clostridium difficile Ribotypes and Severe Disease Presentation and Severe CDI Outcomes ${ }^{a}$

\begin{tabular}{|c|c|c|c|c|}
\hline \multirow[b]{2}{*}{ Model } & \multicolumn{2}{|c|}{ Severe CDI Presentation } & \multicolumn{2}{|c|}{ Severe CDI Outcome } \\
\hline & aOR & $P$ Value & aOR & $P$ Value \\
\hline \multicolumn{5}{|l|}{ Model 1} \\
\hline \multicolumn{5}{|l|}{ Ribotype 027 analysis } \\
\hline Ribotype 027 & $2.24(1.53-3.29)$ & $<.0001$ & $1.71(1.02-2.85)$ & $<.042$ \\
\hline Age & $1.02(1.01-1.03)$ & $<.0001$ & & \\
\hline PPI & $1.74(1.22-2.48)$ & .0020 & & \\
\hline Narcotics & $1.98(1.04-3.79)$ & .037 & & \\
\hline Community-acquired CDI & $0.51(0.33-0.77)$ & .0012 & $0.49(0.26-0.92)$ & .027 \\
\hline Continued use of non-CDI antibiotics & & & $5.35(3.00-9.55)$ & $<.0001$ \\
\hline Treatment of CDI with oral metronidazole & & & $0.30(0.18-0.51)$ & $<.0001$ \\
\hline \multicolumn{5}{|l|}{ Model 2} \\
\hline \multicolumn{5}{|l|}{ Multiple ribotype analysis } \\
\hline Ribotype 027 & $2.01(1.35-2.97)$ & .0005 & & \\
\hline Ribotype 014-020 & $0.57(0.34-0.94)$ & .0027 & $0.26(0.10-0.68)$ & .0060 \\
\hline Age & $1.02(1.01-1.04)$ & $<.0001$ & & \\
\hline PPI & $1.75(1.23-2.49)$ & .0020 & & \\
\hline Narcotics & $1.98(1.04-3.77)$ & .038 & & \\
\hline Community-acquired CDI & $0.50(0.33-0.76)$ & .0012 & $0.46(0.24-0.86)$ & .015 \\
\hline Continued use of non-CDI antibiotics & & & $5.72(3.20-10.2)$ & $<.0001$ \\
\hline Treatment of CDI with oral metronidazole & & & $0.30(0.18-0.52)$ & $<.0001$ \\
\hline \multicolumn{5}{|l|}{ Model 3} \\
\hline \multicolumn{5}{|c|}{ Severe disease presentation and severe CDI outcomes } \\
\hline Severe CDI presentation & & & $4.19(2.46-8.07)$ & $<.00001$ \\
\hline Ribotype 014-020 & & & $0.34(0.13-0.90)$ & .030 \\
\hline Continued use of non-CDI antibiotics & & & $4.45(2.46-8.07)$ & $<.0001$ \\
\hline Treatment of CDI with oral metronidazole & & & $0.38(0.22-0.66)$ & .0006 \\
\hline
\end{tabular}

NOTE. CDI, Clostridium difficile infection; aOR, adjusted odds ratio; PPI, proton pump inhibitor.

${ }^{a}$ Model 1 evaluated the role of ribotype 027 compared with all other ribotypes. Model 2 evaluated all ribotypes independently. Model 3 incorporated severe CDI presentation as an explanatory variable for severe CDI outcomes. 
TABLE 3

Proportion of Patients with Severe Disease Presentation and Severe Disease Outcomes by Ribotype

\begin{tabular}{lcc}
\hline Ribotype & Severe CDI presentation & Severe CDI outcome \\
\hline $027(\mathrm{n}=170)$ & 54.7 & 18.9 \\
$014-020(\mathrm{n}=118)$ & 22.9 & 4.2 \\
FP11 $(\mathrm{n}=70)$ & 31.4 & 8.6 \\
$078-126(\mathrm{n}=42)$ & 21.4 & 9.5 \\
$001(\mathrm{n}=35)$ & 42.9 & 8.6 \\
FP24 $(\mathrm{n}=35)$ & 37.1 & 22.9 \\
$17(\mathrm{n}=23)$ & 39.1 & 17.4 \\
FP8 $(\mathrm{n}=19)$ & 36.9 & 10.5 \\
$053-163(\mathrm{n}=16)$ & 37.5 & 6.25 \\
FP16 $(\mathrm{n}=16)$ & 35.3 & 11.8 \\
FP9 $(\mathrm{n}=16)$ & 25.0 & 18.8 \\
\hline
\end{tabular}

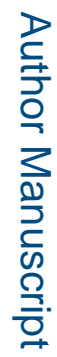

Infect Control Hosp Epidemiol. Author manuscript; available in PMC 2016 January 12. 